\begin{tabular}{|c|l|}
\hline Title & Rhizopus delemar is the proper name for Rhizopus oryzze fumaric-malic acid producers \\
\hline Author(s) & A be, Ayumi; Oda, Y uji; A sano, Kozo; Sone, Teruo \\
\hline Citation & $\begin{array}{l}\text { Mycologia, 99(5), 714 722 } \\
\text { https:/doi.org/L0.3852/my cologia.99.5.714 }\end{array}$ \\
\hline Issue Date & 2007-09 \\
\hline Doc URL & http://hdl.handle.net/2115/38774 \\
\hline Rights & ○ 2007 The Mycological Society of A merica \\
\hline Type & article \\
\hline File Information & sone.pdf \\
\hline
\end{tabular}

Instructions for use 
Mycologia, 99(5), 2007, pp. 714-722.

(C) 2007 by The Mycological Society of America, Lawrence, KS 66044-8897

\section{Rhizopus delemar is the proper name for Rhizopus oryzae fumaric-malic acid producers}

Ayumi Abe

Laboratory of Applied Microbiology, Research Faculty of Agriculture, Hokkaido University, Kita-9 Nishi-9, Kitaku, Sapporo 060-8589, Japan

Yuji Oda

Department of Agricultural and Life Science, Obihiro University of Agriculture and Veterinary Medicine, Obihiro, Hokkaido 080-8555, Japan

Kozo Asano

Teruo Sone ${ }^{1}$

Laboratory of Applied Microbiology, Research Faculty of Agriculture, Hokkaido University, Kita-9 Nishi-9, Kitaku, Sapporo 060-8589, Japan

Abstract: The zygomycete Rhizopus oryzae currently is identified by sporangiophore morphology and growth temperature, but heterogeneity of the species has been reported. We examined the suitability of organic acid production as an effective taxonomic character for reclassification of the species. Strains were divided into two groups, LA (lactic acid producer) and FMA (fumaric-malic acid producers) according to organic acid production. These grouping were confirmed as phylogenetically distinct because analyses of rDNA ITS, lactate dehydrogenase $\mathrm{B}$, actin, translation elongation factor- $1 \alpha$ and genomewide AFLP resolved the same two exclusive clusters, corresponding with the organic acid grouping. Reclassification of strains in the FMA group as $R$. delemar was proposed.

Key words: AFLP, lactic acid, phylogeny, Rhizopus

\section{INTRODUCTION}

Genus Rhizopus includes species often used for fermented foods in eastern and southeastern Asia. Therefore the phylogeny, physiology, genetics and biochemistry of those strains were studied, and many researchers reported new species classified by morphological and physiological characters (Hesseltine 1983, Schipper and Stalpers 1984, Schipper 1984). Schipper and Stalpers (1984) and Schipper (1984) revised the classification of the genus mainly by growth temperature, size of sporangia and sporangiophore and branching of rhizoids and classified all

Accepted for publication 29 July 2007.

${ }^{1}$ Corresponding author. E-mail: sonet@chem.agr.hokudai.ac.jp species of the genus into three groups, stolonifergroup, $R$. oryzae and microsporus-group, with the proposed synonymy of many species. After some additions (Yuan and Jong 1984, Ellis 1985, Weitzman et al 1996), Rhizopus consists of 13 species. Although there were reports of reclassification based on DNADNA hybridization and isozyme analysis (Ellis 1986, Liou et al 2001), Schipper's classification is accepted as the standard of the genus. In the classification by Schipper and Stalpers (1984), Rhizopus oryzae was recognized as a species with intermediate morphology and physiological characters. They investigated 47 strains from 21 species and classified all of them as $R$. oryzae because they fundamentally were indistinguishable.

We recently found a clear relationship between rDNA ITS sequence and acid production in Rhizopus oryzae (Abe et al 2003). In that work, strains of $R$. oryzae were divided clearly into two types, lactic acid producers and fumaric-malic acid producers from the data of organic acid production and the rDNA ITS sequence. In addition, genus-level analysis of rDNA sequences revealed that the degree of diversity between the two groups was similar to that between species (Abe et al 2006). On the other hand, Saito et al (2004) found a relationship between lactic acid production and the $l d h$ genes. $R$. oryzae has two genes for lactate dehydrogenase, $l d h A$ and $l d h B$. Fumaric acid producers lacked the $l d h A$ gene, which was responsible for lactic acid production in lactic acid producers. In addition, the nucleotide sequences of the $l d h B$ gene distinguished the two types. These results indicated the possibility of organic acid production as a physiological category suitable for reclassification of $R$. oryzae, suggesting these two types might be distinct species.

Organic acid production had been studied extensively in $R$. oryzae. Takahashi and Sakaguchi (1925) and Takahashi et al (1926) studied the production of fumaric acid and lactic acid and found that Rhizopus spp. could be divided into fumaric acid producers, lactic acid producers and producers of both fumaric and lactic acid, but they did not comment on the taxonomic importance of acid production. Kitahara and Fukui (1949) indicated that there was no clear relationship between acid production and morphological classification. Inui et al (1965) used acid production as the key character to differentiate $R$. oryzae, $R$. japonicus and $R$. delemar. However, the 
TABLE I. $R$. oryzae strains used in this study with accession numbers of sequence data

\begin{tabular}{|c|c|c|c|c|c|c|}
\hline CBS No. & Former name $^{\mathrm{a}}$ & Proposed name & rDNA ITS & $l d h B$ & $\mathrm{EF}-1 \alpha$ & act-1 \\
\hline 110.17 & R. maydis & R. oryzae & AB181303 & AB281556 & AB281527 & AB281498 \\
\hline $112.07^{\mathrm{T}}$ & R. oryzae & R. oryzae & AB097334 & AB281557 & AB281528 & AB281499 \\
\hline 120.12 & R. delemar & R. delemar & AB181318 & AB281558 & AB281529 & $\mathrm{AB} 281500$ \\
\hline 127.08 & R. nodosus & R. oryzae & AB181304 & AB281559 & AB281530 & AB281501 \\
\hline 128.08 & R. tritici & R. oryzae & $\mathrm{AB} 181305$ & AB281560 & AB281531 & AB281502 \\
\hline 257.28 & R. formosaensis & R. oryzae & AB181311 & AB281561 & AB281532 & AB281503 \\
\hline 258.28 & R. hangchao & R. oryzae & AB181312 & AB281562 & AB281533 & AB281504 \\
\hline 260.28 & R. liquefaciens & R. oryzae & AB181306 & AB281563 & AB281534 & AB281505 \\
\hline 264.28 & R. pseudochinensis & R. oryzae & $\mathrm{AB} 181307$ & AB281564 & AB281535 & AB281506 \\
\hline 266.30 & R. fusiformis & R. oryzae & $\mathrm{AB} 181313$ & AB281565 & AB281536 & $\mathrm{AB} 281507$ \\
\hline 278.38 & R. oryzae & R. delemar & AB097299 & AB281566 & AB281537 & $\mathrm{AB} 281508$ \\
\hline 279.38 & R. sontii & R. delemar & AB181319 & AB281567 & AB281538 & AB281509 \\
\hline 295.31 & R. suinus & $R$. delemar & AB181320 & AB281568 & AB281539 & $\mathrm{AB} 281510$ \\
\hline 321.35 & R. kasanensis & R. oryzae & AB181308 & AB281569 & AB281540 & $\mathrm{AB} 281511$ \\
\hline 330.53 & $R$. boreas & R. oryzae & AB181314 & $\mathrm{AB} 281570$ & AB281541 & $\mathrm{AB} 281512$ \\
\hline 381.52 & R. arrhizus & $R$. oryzae & AB181315 & $\mathrm{AB} 281571$ & AB281542 & AB281513 \\
\hline 385.34 & R. achlamydosporus & R. delemar & AB181321 & AB281572 & AB281543 & AB281514 \\
\hline 386.34 & R. bahrnensis & $R$. delemar & AB181322 & $\mathrm{AB} 281573$ & AB281544 & $\mathrm{AB} 281515$ \\
\hline 387.34 & R. batatas & $R$. oryzae & AB181309 & AB281574 & AB281545 & AB281516 \\
\hline 389.34 & R. chiuniang var. isofermentarius & R. delemar & AB181323 & $\mathrm{AB} 281575$ & AB281546 & $\mathrm{AB} 281517$ \\
\hline 390.34 & $R$. delemar var. minimus & R. delemar & AB181324 & AB281576 & AB281547 & AB281518 \\
\hline 391.34 & R. javanicus & $R$. delemar & AB181325 & $\mathrm{AB} 281577$ & AB281548 & AB281519 \\
\hline 392.95 & R. delemar var. multiplicisporus & R. delemar & AB181326 & $\mathrm{AB} 281578$ & AB281549 & $\mathrm{AB} 281520$ \\
\hline 393.34 & R. peka II & R. delemar & AB181327 & AB281579 & AB281550 & $\mathrm{AB} 281521$ \\
\hline 395.34 & R. semarangensis & R. delemar & AB181316 & AB281580 & AB281551 & AB281522 \\
\hline 395.54 & & $R$. delemar & AB181317 & AB281581 & AB281552 & AB281523 \\
\hline 402.51 & R. javanicus var. kawasakiensis & $R$. delemar & AB181328 & AB281582 & AB281553 & AB281524 \\
\hline 404.51 & R. shanghaiensis & $R$. delemar & AB181329 & AB281583 & AB281554 & $\mathrm{AB} 281525$ \\
\hline 406.51 & R. usamii & R. delemar & AB181330 & AB281584 & AB281555 & AB281526 \\
\hline
\end{tabular}

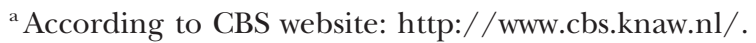

classification was not accepted and the current taxonomy was based on morphological characters. In this study we propose to use organic acid productivity as the key feature to reclassify $R$. oryzae fumaric-malic acid producers as $R$. delemar, with the molecular phylogeny based on sequence data of the rDNA ITS, $l d h B$, EF- $1 \alpha$ and actin as well as genomic AFLP.

\section{MATERIALS AND METHODS}

Strains and growth conditions.-Rhizopus oryzae strains used in this study are listed (TABLE I). All strains were obtained from the Centraalbureau voor Schimmelcultures (CBS, Utrecht, Netherlands). For preservation and serial transfer, potato-glucose agar (Difco, Detroit, Michigan) was used. The medium for DNA preparation was malt extract (malt extract [Difco] $20 \mathrm{~g} / \mathrm{L}$, polypepton [Nihon Pharmaceutical, Tokyo] $1 \mathrm{~g} / \mathrm{L}$, and glucose $20 \mathrm{~g} / \mathrm{L}$ ). Organic acid production was investigated according to Saito et al (2004).

DNA extraction.-Liquid-cultured cells were filtered, airdried, and lyophilized overnight. The genomic DNA of each strain was extracted from the lyophilized cells according to Sone et al (1997).
Amplification and sequencing of $r D N A$ ITS region, ldhB, EF$1 a$ and act1.- rDNA ITS region, $l d h B$ and act1 were amplified and sequenced with primers described respectively in White et al (1990), Saito et al (2004) and Voigt and Wöstemeyer (2001). EF-1 $\alpha$ was amplified and sequenced with listed primers (TABLE II). PCR amplification was performed in $50 \mu \mathrm{L}$ reaction mixture containing $5 \mu \mathrm{L}$ of $10 \times$ PCR buffer, $5 \mu \mathrm{L}$ deoxynucleotide triphosphate $(2 \mathrm{mM}$ each), 10 pmole of each primer, $3.5 \mu \mathrm{L} \mathrm{MgCl}_{2}$ solution (25 mM), $2.5 \mathrm{U}$ AmpliTaq DNA polymerase (Applied Biosystems, Foster City, California) and $100 \mathrm{ng}$ of template DNA of each strain. The reaction conditions were: initial denaturation at $94 \mathrm{C}$ for $2 \mathrm{~min}, 35$ cycles of denaturation at $94 \mathrm{C}$ for $15 \mathrm{~s}$, annealing at $55 \mathrm{C}$ for $30 \mathrm{~s}$, extension at $72 \mathrm{C}$ for $1 \mathrm{~min}$ and a final $5 \mathrm{~min}$ of elongation at $72 \mathrm{C}$ in a Model 9700 Thermal Cycler (Applied Biosystems), except that the annealing temperature for actin primers was $50 \mathrm{C}$. PCR products were purified with Microspin S-300HR (Amersham Biosciences, Piscataway, New Jersey). Sequencing reactions were performed with a BigDye ${ }^{\mathrm{TM}}$ Terminator Cycle Sequence Ready Reaction Kit (Applied Biosystems) and analyzed by ABI PRISM 3100 Genetic Analyzer or ABI PRISM 310 Genetic Analyzer (Applied Biosystems). Sequence alignments were performed with Clustal X (Thomp- 
TABLE II. Primers used for the amplification and sequencing of EF- $1 \alpha$

\begin{tabular}{llll}
\hline \hline Primer & \multicolumn{1}{c}{ Usage $^{\mathrm{a}}$} & \multicolumn{1}{c}{ Sequence $\left(5^{\prime}\right.$ to $\left.3^{\prime}\right)$} & \multicolumn{1}{c}{ Reference } \\
\hline MEF-10 & Amp, Seq & GTTGTCATCGGTCACGTCGATTC & This study \\
MEF-20 & Seq & GGATACCACCAAGTGGTCCG & This study \\
MEF-30 & Seq & GTCGAAATGCACCACGAAAC & This study \\
MEF-50 & Seq & GGGTTTCGTGGTGCATTTCG & This study \\
MEF-60 & Seq & CGGACCACTTGGTGGTATCC & This study \\
MEF-4 & Amp, Seq & ATGACACCRACAGCGACGGTTTG & O'Donnell et al (2001) \\
\hline
\end{tabular}

${ }^{\mathrm{a}} \mathrm{Amp}=$ PCR amplification; Seq = sequencing.

son et al 1997). Parsimony analysis was performed with PAUP* 4.0b10 (Swofford 2002). Sequence data was deposited in the DDBJ/EMBL/GenBank database. (Accession numbers listed in TABLE I.)

AFLP analysis._-Amplified fragment length polymorphism (AFLP) analysis was performed with an AFLP Microbial Fingerprinting Kit (Applied Biosystems) according to the manufacturer's protocol. AFLP samples were prepared with 10 combinations of selective primers; $E c o \mathrm{R} \mathrm{I}+\mathrm{A}$ and $M s e \mathrm{I}+$ $\mathrm{T}, E c o \mathrm{R} \mathrm{I}+\mathrm{C}$ and $M s e \mathrm{I}+\mathrm{A}, E c o \mathrm{R} \mathrm{I}+\mathrm{C}$ and $M s e \mathrm{I}+\mathrm{C}, E c o \mathrm{R} \mathrm{I}$ $+\mathrm{C}$ and $M s e \mathrm{I}+\mathrm{G}, E c o \mathrm{R} \mathrm{I}+\mathrm{C}$ and $M s e \mathrm{I}+\mathrm{T}, E c o \mathrm{R} \mathrm{I}+\mathrm{G}$ and $M s e \mathrm{I}+\mathrm{C}, E c o \mathrm{R} \mathrm{I}+\mathrm{G}$ and $M s e \mathrm{I}+\mathrm{G}, E c o \mathrm{R} \mathrm{I}+\mathrm{G}$ and $M s e \mathrm{I}+$ $\mathrm{T}, E c o \mathrm{R} \mathrm{I}+\mathrm{T}$ and $M s e \mathrm{I}+\mathrm{C}$, and $E c o \mathrm{R} \mathrm{I}+\mathrm{T}$ and $M s e \mathrm{I}+\mathrm{T}$. Amplified samples were analyzed with an ABI PRISM 3100 genetic analyzer and Genescan software (Applied Biosystems). Electrophoresis results were analyzed and converted to binary data with Genotyper software (Applied Biosystems). The binary data were converted with Windist (Yap and Nelson 1996) to a distance matrix, and the data were subjected to clustering using Neighbor software of PHYLIP (Felsenstein 2004). Bootstrap analysis was performed with Winboot (Yap and Nelson 1996) software with 1000 iterations. DNA sequence alignments, AFLP matrix and trees were deposited in TreeBase (study accession number $=\mathrm{S} 1869$ ).

\section{RESULTS}

$R$. oryzae strains were chosen from the strains used in the study by Schipper and Stalpers (1984), which is the current classification of the genus. This enabled us to compare directly the results of our study with the morphological classification.

Production of organic acids and ethanol of all $R$. oryzae strains was determined as listed (TABLE III). Thirteen strains produced mainly lactic acid and other strains produced fumaric acid and malic acid. The amounts of malic acid and ethanol produced in the latter group were higher than those of the former. From these data strains were divided into two groups, LA (lactic acid producer) and FMA (fumaric acid and malic acid producer) (TABLE III).

rDNA ITS sequences of all strains were classified into four types (TABLE IV). These sequences were subjected to parsimony analysis (FIG. 1). They grouped into two clusters. Members of each cluster corresponded to that of each organic acid production group (TABLE III).

Further cluster analyses were performed with the DNA sequences of lactate dehydrogenase B $(l d h B)$, actin $(a c t 1)$ and translation elongation factor- $1 \alpha(\mathrm{EF}-$ $1 \alpha$ ) (FIG. 2). Larger diversity of sequence was detected in these three phylogenetic trees than in the rDNA ITS tree. Two large clusters corresponding to the LA and FMA clusters in rDNA ITS tree were resolved with high bootstrap values, except the tree based on $l d h B$. In the $l d h B$ tree CBS391.34 was located outside the major clusters, but the bootstrap value for the FMA group including CBS391.34 was the highest, at 100, indicating a strong relationship among CBS391.34 and other FMA group strains.

Two subclusters were detected in each of the large clusters in the EF-1 $\alpha$ tree. These subclusters corresponded to the groups of the ITS sequence A, B, C and D. In the other two trees at least one subcluster consisted of strains with a different ITS sequence. In the $l d h B$ tree all strains of the FMA group except CBS 391.34 formed a cluster. In the act 1 tree as well, three strains with ITS sequence B clustered with the ITS sequence A strains (FIG. 2).

AFLP analysis was performed to infer phylogenetic relationships at the whole genome level. A total of 301 polymorphic markers, selected from bands amplified with 10 pairs of selective primers, were used for the analysis. The neighbor joining tree based on AFLP data is shown (FIG. 3). Two large clusters corresponding to the LA group and FMA group were resolved with high bootstrap values. Subclusters A, B, C and D also were resolved.

\section{DISCUSSION}

The aim of this study was to evaluate organic acid production as the key to reclassify $R$. oryzae. Organic acid production of $R$. oryzae was reported in recent studies to divide the species into two groups, lactic acid producers and fumaric-malic acid producers, but strains used in those studies did not contain authentic 
TABLE III. Production of organic acids and ethanol by the Rhizopus oryzae strain used

\begin{tabular}{|c|c|c|c|c|c|c|}
\hline Group & CBS No. & Lactic acid $\left(\mathrm{mg} \mathrm{mL}^{-1}\right)$ & $\begin{array}{l}\text { Fumaric acid } \\
\left(\mathrm{mg} \mathrm{mL}^{-1}\right)\end{array}$ & $\begin{array}{l}\text { Malic acid } \\
\left(\mathrm{mg} \mathrm{mL}^{-1}\right)\end{array}$ & $\begin{array}{c}\text { Ethanol } \\
\left(\mathrm{mg} \mathrm{mL}^{-1}\right)\end{array}$ & ITS type \\
\hline LA & 110.17 & 43.2 & 0.0 & $<0.1$ & 4.7 & A \\
\hline LA & $112.07^{\mathrm{T}}$ & 42.9 & 0.0 & $<0.1$ & 3.6 & A \\
\hline LA & 127.08 & 42.9 & 0.0 & $<0.1$ & 4.6 & A \\
\hline LA & 128.08 & 42.9 & 0.0 & 0.4 & 3.7 & A \\
\hline LA & 257.28 & 39.5 & 0.0 & 0.3 & 5.6 & B \\
\hline LA & 258.28 & 40.2 & 0.0 & $<0.1$ & 3.6 & B \\
\hline LA & 260.28 & 41.9 & 0.0 & $<0.1$ & 5.2 & A \\
\hline LA & 264.28 & 45.4 & 0.0 & 0.5 & 5.9 & A \\
\hline LA & 266.30 & 40.8 & 0.0 & $<0.1$ & 2.9 & B \\
\hline LA & 321.35 & 43.4 & 0.0 & 0.3 & 4.5 & A \\
\hline LA & 330.53 & 34.6 & 0.0 & 0.8 & 7.1 & B \\
\hline LA & 381.52 & 16.4 & 0.0 & 1.9 & 13.4 & $\mathrm{~B}$ \\
\hline LA & 387.34 & 33.9 & 0.0 & 0.6 & 5.9 & A \\
\hline FMA & 120.12 & 0.0 & 0.8 & 11.7 & 13.1 & $\mathrm{D}$ \\
\hline FMA & 278.38 & 0.0 & 3.6 & 10.9 & 14.1 & $\mathrm{D}$ \\
\hline FMA & 279.38 & 0.0 & 1.3 & 8.5 & 15.0 & $\mathrm{D}$ \\
\hline FMA & 295.31 & 0.0 & 0.7 & 9.0 & 14.7 & $\mathrm{D}$ \\
\hline FMA & 385.34 & 0.0 & 1.1 & 3.2 & 17.0 & $\mathrm{D}$ \\
\hline FMA & 386.34 & 0.0 & 0.9 & 6.2 & 15.7 & $\mathrm{D}$ \\
\hline FMA & 389.34 & 0.0 & 1.3 & 9.0 & 14.6 & $\mathrm{D}$ \\
\hline FMA & 390.34 & 0.0 & 1.1 & 10.4 & 13.6 & $\mathrm{D}$ \\
\hline FMA & 391.34 & 0.0 & 0.5 & 5.7 & 15.4 & $\mathrm{D}$ \\
\hline FMA & 392.95 & 0.0 & 1.0 & 11.2 & 11.7 & $\mathrm{D}$ \\
\hline FMA & 393.34 & 0.0 & 0.8 & 8.7 & 16.1 & $\mathrm{D}$ \\
\hline FMA & 395.34 & 0.0 & 0.4 & 9.6 & 17.0 & $\mathrm{C}$ \\
\hline FMA & 395.54 & 0.0 & $<0.1$ & 3.2 & 18.3 & $\mathrm{C}$ \\
\hline FMA & 402.51 & 0.0 & 4.3 & 12.0 & 12.6 & $\mathrm{D}$ \\
\hline FMA & 404.51 & 0.0 & 0.8 & 11.1 & 14.4 & $\mathrm{D}$ \\
\hline FMA & 406.51 & 0.0 & 0.6 & 6.1 & 17.7 & $\mathrm{D}$ \\
\hline
\end{tabular}

type strains so they could not make detailed taxonomic delimitations (Abe et al 2003, Oda et al 2003, Saito et al 2004, Abe et al 2006). In this study we used the strains on which the current classification is based to evaluate species limits.

These strains were classified into two groups, LA (lactic acid producers) and FMA (fumaric-malic acid producers) (TABLE III) as shown by Oda et al (2003) and Saito et al (2004) with a different set of strains. FMA strains did not produce lactic acid at all. Saito et al (2004) found that the lack of lactic acid was due to the absence of the $l d h A$ gene, which is responsible for its production. On the other hand LA strains did not produce fumaric acid at all, but this does not indicate the lack or mutation of the gene responsible for fumaric acid production because fumaric acid is produced as an intermediate of the TCA cycle (Saito et al 2004).

The lactic acid production of the LA strains and ethanol production of FMA strains was about twice as much as that of strains used by Saito et al (2004). This could be due to a difference in culture conditions. We tried to cultivate them under the same conditions, but there might have been less aeration in our case because both metabolites are produced from pyruvate anaerobically. Consequently the differentiation of strains based on organic acid production became clearer than in the study of Saito et al (2004).

Organic acid production once was used as a key taxonomic character within Rhizopus by Inui et al (1965), but this was not accepted in other works, probably because these physiological characteristics are easily affected by environmental factors, such as media and aeration. In this study it was confirmed strongly that the grouping of $R$. oryzae strains into LA and FMA was phylogenetically robust from the sequence of the four independent genes, rDNA-ITS, $l d h B, a c t 1$, and translation elongation factor- $1 \alpha$ as well as the genomewide AFLP analysis. Bootstrap values of the clusters for LA groups and FMA groups were more than $70 \%$. This also strengthens the opinion by Saito et al (2004) that the loss of lactic acid production in FMA strains was not due to a mutation of LA strains. 
TABLE IV. Four types of rDNA ITS sequence found in $R$. oryzae strains

\begin{tabular}{ccccccc}
\hline \hline & \multicolumn{7}{c}{ Position in D type sequence } \\
\cline { 2 - 7 } Sequence type & 52 & 54 & 174 & 414 & 517 & 559 \\
\hline A & - & - & T & G & T & T \\
B & - & - & C & G & T & T \\
C & - & - & C & A & C & C \\
D & C & T & C & A & C & C \\
\hline
\end{tabular}

In each organic acid production group subclusters corresponding to the ITS sequence type were detected. However all four subclusters corresponding to four rDNA ITS types were found only from the trees of EF- $1 \alpha$ and AFLP. This might indicate the occurrence of recombination between strains of the

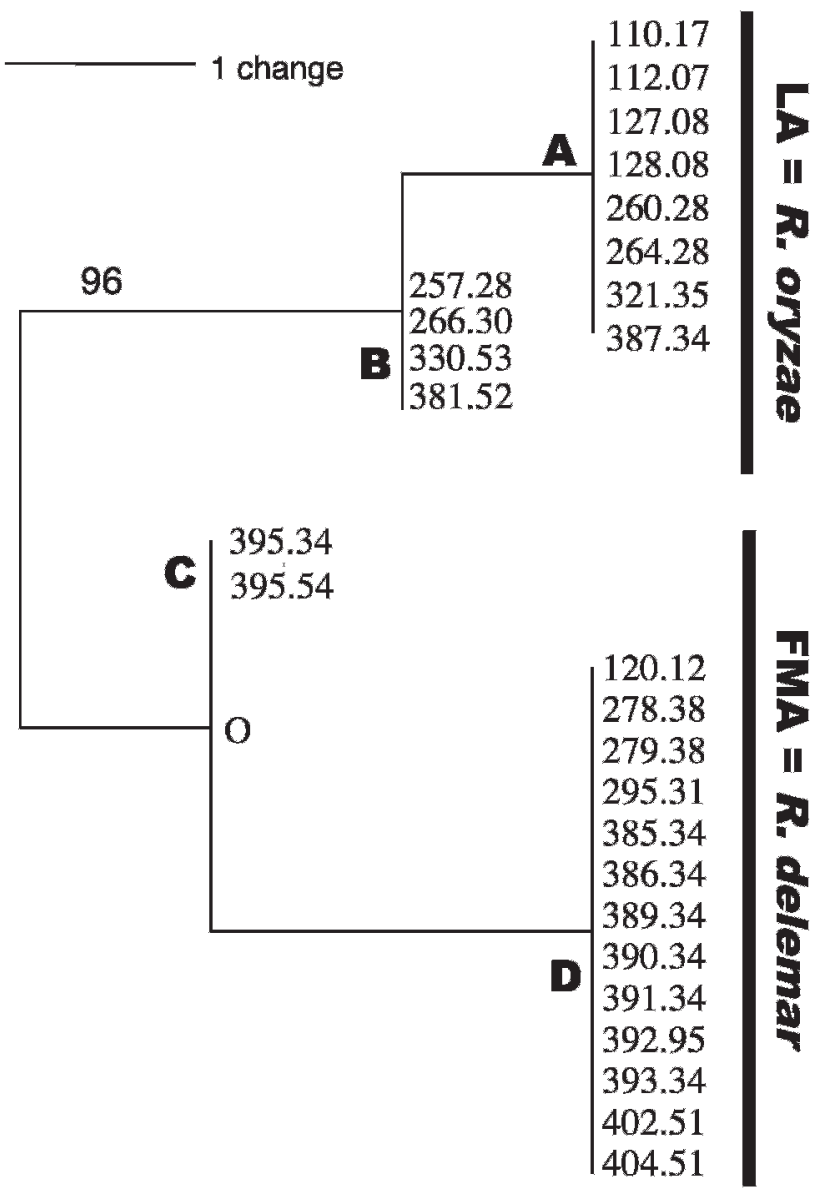

FIG. 1. Single most parsimonious tree based on rDNA ITS sequence of $R$. oryzae and $R$. delemar. Taxa are indicated with CBS culture numbers. Gaps were treated as fifth base. Tree is written by treating the data of C and D type sequences as outgroup cluster, indicated by the letter $\mathrm{O}$. Tree parameters: length $=6, \mathrm{CI}=1.0$. The bootstrap value is for the $\mathrm{A}$ and $\mathrm{B}$ cluster of sequences. Sequence types are indicated with A, B, C and D. Organic acid production groups are shown at right. two ITS types within each organic acid producers in the past. The limit of species accordingly can be made at the organic acid groupings if we apply genealogical concordance phylogenetic species recognition (GCPSR, Taylor et al 2000) in this situation.

On the other hand, considering that the occurrence of $\mathrm{A}+\mathrm{B}$ clade in only the act1 tree might be due to ancestral polymorphism (Takahata and Nei 1985 ) or homoplasy rather than ongoing gene flow, two cryptic species corresponding to groups A and B can be recognized. However, the fact that we could not detect any morphological or physiological polymorphism between groups A and B prevented us from placing the species limit at this level.

Ellis (1985) proposed to reclassify $R$. oryzae, $R$. delemar and its varieties, $R$. chungkuoensis var. isofermentarius, $R$. javanicus var. kawasakiensis, $R$. arrhizus and its varieties, and $A$. rouxii into three varieties of $R$. arrhizus, (i.e. var. arrhizus, var. delemar, and var. rouxii) based on DNA-DNA hybridization data. Compared with our AFLP data, his proposed varieties, $R$. arrhizus var. arrhizus and var. delemar, correspond respectively to LA group and FMA group in our study, although the strains in his study were not the same as those used in ours. His basis for the proposal of "variety" instead of "species" were small morphological difference of sporangiospore and intermediate DNA complementarity (65\%) between two varieties of $R$. arrhizus, in comparison with the higher rate $(>90 \%)$ within the same variety and the lower rate (18\%) among species $R$. arrhizus and $R$. microsporus. Shipper and Stalpers (1984) proposed three groups of Rhizopus species, the stolonifer-group, microsporusgroup and oryzae. The significance of this grouping recently was confirmed by Frye and Reinhardt (1993) and Abe et al (2006), using respectively $\mathrm{G}+\mathrm{C}$ content and rDNA ITS sequence. The lower DNA complementarity between $R$. arrhizus and $R$. microsporus corresponds with the genetic distance between the species. Further the intermediate complementarity between the two varieties of $R$. arrhizus is enough for them to be divided into separate species.

Another important issue for species recognition is mating. Schipper and Stalpers (1984) reported that zygospores were obtained in some crosses between $R$. 
oryzae strains, CBS346.36 × 112.07; 127.08; 110.17; $148.22 ; 257.28 ; 264.28 ; 266.30 ; 329.47 ; 382.52$; and 285.55. In this study strains except 346.36, 148.22, $329.47,382.52,285.55$ were shown to be the LA type, and no strains identified as the FMA type in this study were included in the list of fertile strains. This suggests that all fertile crosses observed by Schipper and Stalpers (1984) were between LA type strains, and no crosses between LA and FMA have been observed yet. This supports the idea that LA and FMA strains represent two species.

In conclusion, we propose to separate FMA group strains into separate species. To our knowledge Rhizopus delemar (Boidin)Wehmer and Hanzawa reported in Hanzawa (1912) is the first name given to the strains of the FMA group, thus it is the name we propose for this species. $R$. oryzae should remain the name of LA group strains because the current type culture of $R$. oryzae is included in this group. The key feature for the separation is organic acid production, but one can identify $R$. delemar by sequencing of rDNA-ITS region based on the different nucleotides (TABLE IV). The description for $R$. oryzae by Schipper (1984) should remain as that for the common feature of $R$. oryzae and $R$. delemar, and thus these species will form the third group in the genus Rhizopus, the oryzae-delemar group.

It was impossible for us to assign the new species name for $R$. tonkinensis, $R$. japonicus, $R$. norwegicus, $R$. chunkuoensis and $R$. thermosus among the species listed in Shipper (1984) due to the unavailability of type culture for those species. However, we were able to assign the new species name to these, with the exception of $R$. norwegicus, using the rDNA ITS sequence data of the culture stocked in our laboratory, some of which were provided by the original identifiers, R. tonkinensis AHU6559 and AHU6560 (GenBank accession Nos. AB097338 and AB097339), R. japonicus AHU6524 and AHU6525 (GenBank accession Nos. AB097346 and AB097347), R. chunkuoensis AHU6513 (GenBank accession Nos. AB097365) and R. thermosus AHU6557 andAHU6558 (GenBank accession Nos. AB097357 and AB097358).

We have no clear explanation for the factors that led to the speciation of these two groups. Many strains of $R$. oryzae including some strains used in this study were isolated from fermented foods or their starters. One guess is that human activity, such as the selection of appropriate strains for fermentation foods, might have played a role.

\section{DESCRIPTION OF SPECIES}

Rhizopus delemar (Boidin) Wehmer \& Hanzawa, Mykol. Zentralbl. 1:77. 1912 emend. A Abe, Y Oda, $\mathrm{K}$ Asano and $\mathrm{T}$ Sone $\equiv$ Mucor delemar Boidin,

$=R$. sontii

$=R$. suinus

$=R$. aclhlamydosporus

$=R$. bahrensis

$=R$. chiuniang var. isofermentarius

$=R$. delemar var. minimus

$=R$. javanicus

$=R$. peka

$=R$. semarangensis

$=R$. javanicus var. kawasakiensis

$=R$. usamii

$=R$. shanghaiensis

$=R$. delemar var. multiplicisporus

$=R$. chunkuoensis

Sporangiophores up to $1500 \mu \mathrm{m}$ long, with local swellings, brown, single or aggregated in small groups. Sporangia grayish black, powdery, up to $200 \mu \mathrm{m}$ diam. Columellae ellipsoidal on a truncate base, mouse-gray or brownish. Sporangiospores angular, subglobose to ellipsoidal, with ridges on the surface, up to $12 \mu \mathrm{m}$ long. No growth at $45 \mathrm{C}$, growth at 40 C. Does not produce lactic acid from a carbon source in liquid rotary culture.

Cultures examined: JAPAN, isolated by J. Hanzawa CBS $120.12=$ former type culture of $R$. delemar, FRANCE, isolated by $\mathrm{H}$. Boulard CBS 278.38; INDIA, CBS279.38 = former type culture of $R$. sontii; GERMANY, isolated from pig CBS 295.31 = former type culture of $R$. suinus; JAPAN, CBS $385.34=$ former type culture of $R$. achlamydosporus; CBS 386.34 = former type culture of $R$. bahrnensis; CBS $389.34=$ former type culture of $R$. chiuniang var. isofermentarius; CBS $390.34=$ former type culture of $R$. delemar var. minimus; CBS $391.34=$ former type culture of $R$. javanicus, CBS $393.34=$ former type culture of $R$. peka; CBS 395.34 = former type culture of $R$. semarangensis; CBS $402.51=$ former type culture of $R$. javanicus var. kawasakiensis; CBS $406.51=$ former type culture of $R$. usamii; CHINA, CBS $404.51=$ former type culture of $R$. shanghaiensis; UNITED STATES. GEOGIA: Chamblee, isolated from man, Mucor mycosis of diabetic patient, by B. West CBS 395.54; CBS 392.95 = former type culture of $R$. delemar var. multiplicisporus.

Rhizopus oryzae Went. \& Prinsen Geerl., Verh. K. Akad. Wet., Sect. 2, 4:16. 1895 emend. A Abe, Y Oda, K Asano and T Sone

$=$ ? R. arrhizus

$=R$. maydis

$=R$. nodosus

$=R$. tritici

$=R$. formosaenensis

$=R$. hangchao

$=R$. liquefaciens

$=R$. pseudochinensis

$=R$. fusiformis

$=R$. kasanensis

$=R$. boreas 


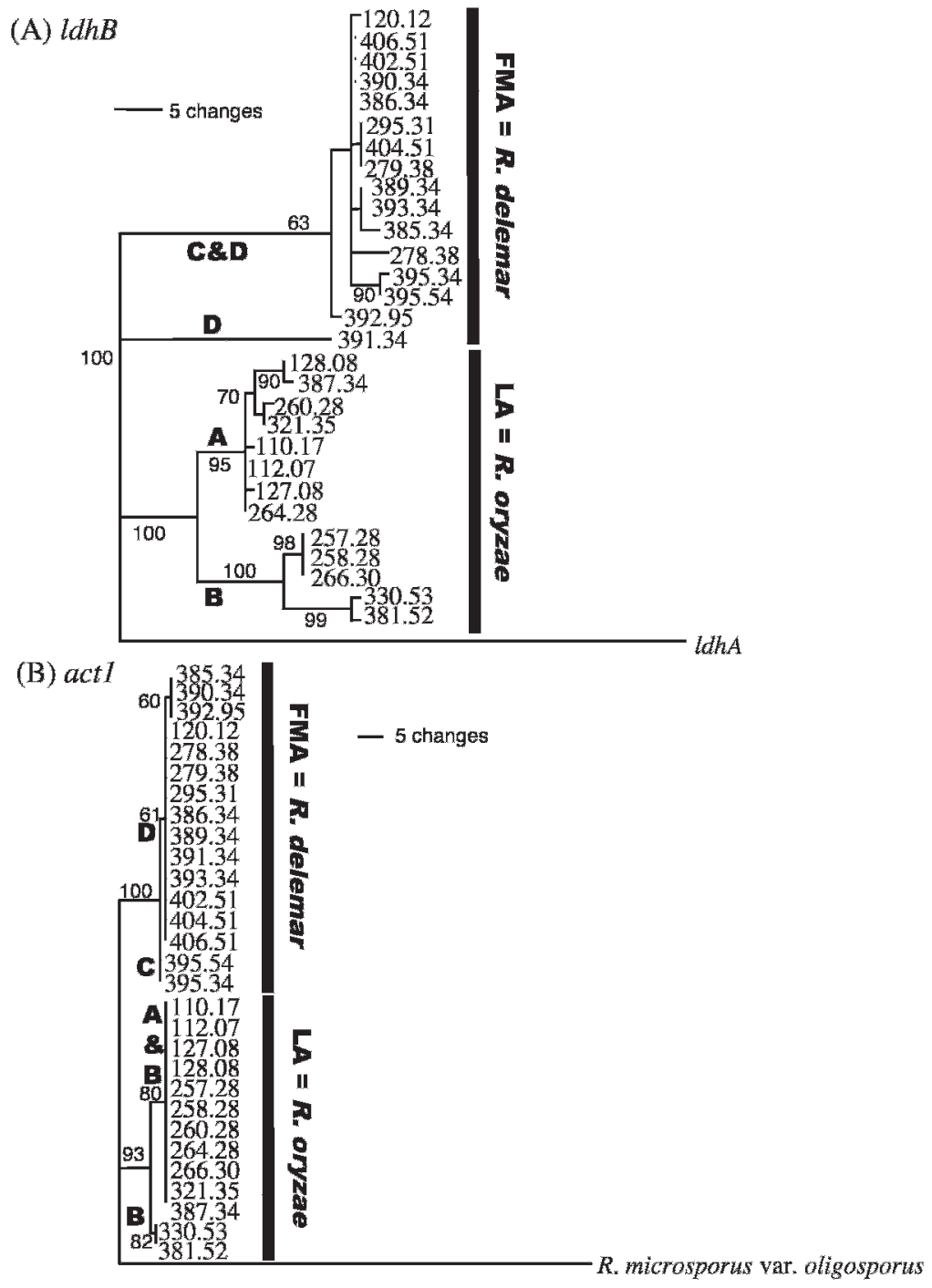

(C) $\mathrm{EF}-1 \alpha$

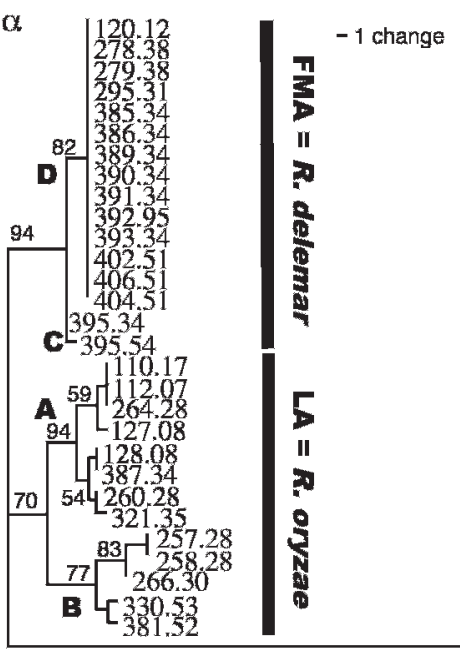

FIG. 2. Parsimonious trees based on DNA sequences of $l d h B$, act 1 and EF- $1 \alpha$ of $R$. oryzae and $R$. delemar. Taxa are indicated with CBS culture numbers. Bootstrap values are shown as percentage calculated from 500 iterations. rDNA-ITS sequence types are indicated by A, B, C or D. Organic acid production groups are shown at the right of taxa. A. One of 64 most parsimonious trees based on ldhB sequence. ldhA sequence of $R$. oryzae NRRL 395 (accession No. AF226154, Skory 2000) was used as 


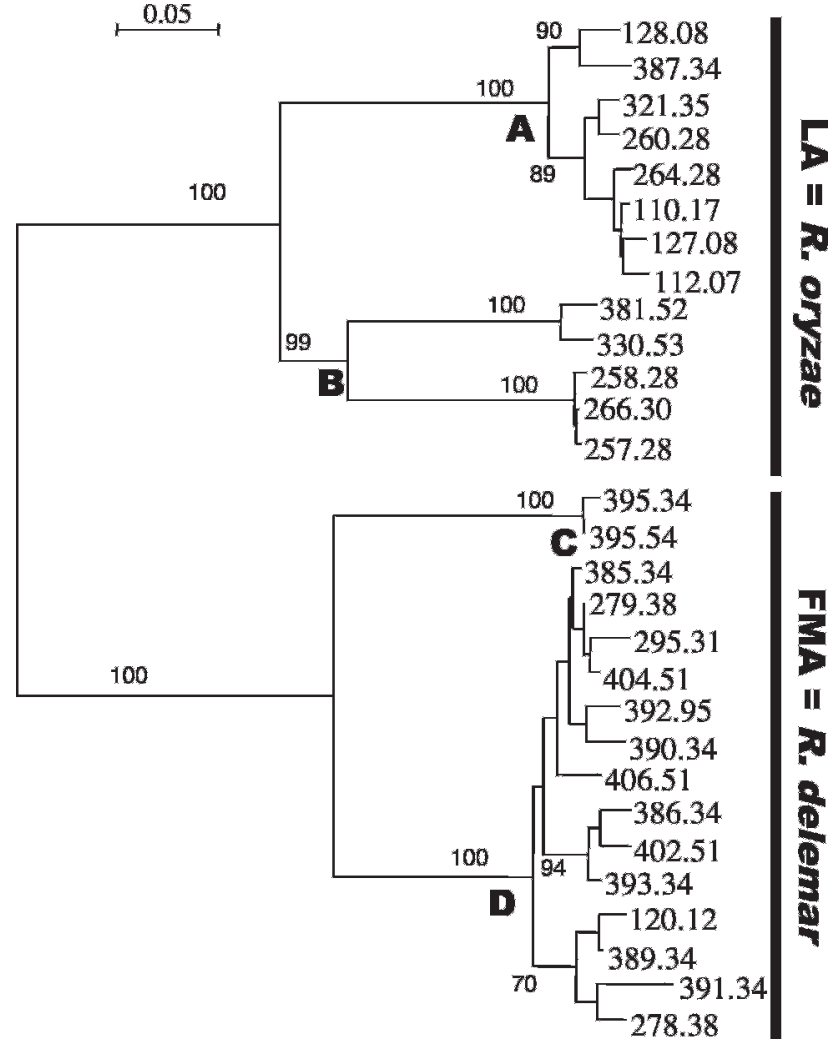

FIG. 3. Neighbor joining tree based on AFLP data for $R$. oryzae and $R$. delemar. Taxa are indicated with CBS culture numbers. Bootstrap values are shown in percentage calculated from 1000 iterations. rDNA-ITS sequence types are indicated by A, B, C or D. Organic acid production groups are shown at right of taxa.

$=R$. batatas

$=R$. tonkinensis

$=R$. japonicus

$=R$. thermosus

Sporangiophores on stolons up to $1500 \mu \mathrm{m}$ long, with local swellings, brown, single or aggregated in small groups. Sporangia grayish black, powdery, up to $200 \mu \mathrm{m}$ diam. Columellae ellipsoidal on a truncate base, mouse-gray or brownish. Sporangiospores angular, subglobose to ellipsoidal, with ridges on the surface, up to $10 \mu \mathrm{m}$ diam. No growth at $45 \mathrm{C}$, growth at 40 C. Produces lactic acid from a carbon source in liquid rotary culture.

Cultures examined: NETHERLANDS, isolated by F.A.F.C. Went CBS $112.07=$ former type culture of $R$. oryzae; SWITZERLAND, isolated by A. Lendner CBS110.17 $=$ former type culture of $R$. maydis; CBS $127.08=$ former authentic culture of $R$. nodosus; CHINA, isolated by K. Saito CBS128.08 $=$ former authentic culture of $R$. tritici; TAIWAN, isolated by R. Nakazawa CBS 257.28 = former type culture of $R$. formosaensis; CHINA, isolated by $\mathrm{M}$. Yamazaki CBS 258.28 = former type culture of $R$. hangchao; CHINA, isolated by M. Yamazaki CBS 260.28 = former type culture of $R$. liquefaciens; CHINA, isolated by M. Yamazaki CBS 264.28 = former type culture of $R$. pseudochinensis; UNITED STATES, isolated by B.B. Kanouse CBS $266.30=$ former type culture of $R$. fusiformis; CBS 321.35 former $R$. kasanensis; JAPAN, CBS 330.53 = former type culture of $R$. boreas; UNITED STATES, isolated by H.C. Murray CBS 381.52; JAPAN, isolated by R. Nakazawa CBS $387.34=$ former type culture of $R$. batatas.

\section{ACKNOWLEDGMENTS}

This work was supported in part by the Special Coordination Funds for Promoting Science and Technology (Leading Research Utilizing the Potential of Regional Science and Technology) of the Ministry of Education, Culture, Sports, Science, and Technology of the Japanese Government. The authors are grateful to Dr. C.D. Skory (USDAARS) and Dr. A. Balajee for kind assistance in the preparation of this manuscript.

\section{LITERATURE CITED}

Abe A, Sone T, Sujaya I-N, Saito K, Oda Y, Asano K, Tomita F. 2003. rDNA ITS sequence of Rhizopus oryzae: its application to classification and identification of lactic acid producers. Biosci Biotechnol Biochem 67:17251731.

— Oda Y, Asano K, Sone T. 2006. The molecular phylogeny of the genus Rhizopus based on rDNA sequences. Biosci Biotechnol Biochem 70:2387-2393.

Ellis JJ. 1985. Species and varieties in the Rhizopus arrhizusRhizopus oryzae group as indicated by their DNA complementarity. Mycologia 77:243-247.

__- 1986. Species and varieties in the Rhizopus microsporus group as indicated by their DNA complementarity. Mycologia 78:508-510.

Felsenstein J. 2004. PHYLIP (Phylogeny Inference Package). Version 3.6. Seattle: Department of Genome Sciences, University of Washington. Distributed by the author.

Frye CB, Reinhardt DJ. 1993. Characterization of groups of the zygomycete genus Rhizopus. Mycopathologia 124: 139-147.

outgroup. Tree parameters: length $=138, \mathrm{CI}=0.899$. B. Single most parsimonious tree based on act 1 sequence. Sequence of R. microsporus var. oligosporus CBS 338.62 (accession No. AJ287197, Voigt et al 2001) was used as outgroup. Tree parameters: length $=112, \mathrm{CI}=0.982$. C. One of eight most parsimonious trees based on EF-1 $\alpha$ sequence. Sequence of $R$. microsporus var. oligosporus CBS 338.62 (accession No. AF157288, O'Donnell et al 2001) was used as outgroup. Tree parameters: length = 96, $\mathrm{CI}=0.854$. 
Hanzawa J. 1912. Zur Morphologie und Physiologie von Rhizopus Delemar, dem Pilz des neuren AmyloVerfahrens. Mykol Zentbl 1:76-91.

Hesseltine CW. 1983. Microbiology of oriental fermented foods. Ann Rev Microbiol 37:575-601.

Inui T, Takeda Y, Iizuka H. 1965. Taxonomical studies on genus Rhizopus. J Gen Appl Microbiol 11. Suppl.

Kitahara K, Fukui S. 1949. Rhizopus zoku kin no nyuusan seiseinou ni tsuite (Study on the lactic acid productivity of genus Rhizopus). Hakko-Kogaku Zasshi 27:218-222. (In Japanese).

Liou G-Y, Chen C-C, Yuan G-F, Chien C-Y. 2001. A taxonomic study of the genus Rhizopus by isozyme patterns. Nov Hedwig 72:231-239.

Oda Y, Yajima Y, Kinoshita M, Ohnishi M. 2003. Differences of Rhizopus oryzae strains in organic acid synthesis and fatty acid composition. Food Microbiol 20:371-375.

O’Donnell K, Lutzoni FM, Ward TJ, Benny GL. 2001. Evolutionary relationships among mucoralean fungi (Zygomycota): evidence for family polyphyly on a large scale. Mycologia 93:286-296.

Saito K, Saito A, Ohnishi M, Oda Y. 2004. Genetic diversity in Rhizopus oryzae strains as revealed by the sequence of lactate dehydrogenase genes. Arch Microbiol 182:30-36.

Schipper MAA. 1984. A revision of the genus Rhizopus 1. The Rh. stolonifer-group and Rh. oryzae. Stud Mycol 25:1-19. -, Stalpers JA. 1984. A revision of the genus Rhizopus II. The Rh. microsporus-group. Stud Mycol 25:20-34.

Skory CD. 2000. Isolation and expression of lactate dehydrogenase genes from Rhizopus oryzae. Appl Environ Microbiol 66:2343-2348.

Sone T, Abe T, Yoshida N, Suto M, Tomita F. 1997. DNA fingerprinting and electrophoretic karyotyping of Japanese isolates of rice blast fungus. Ann Phytopathol Soc Jpn 63:155-163.

Swofford DL. 2002. PAUP*: phylogenetic analysis using parsimony (*and other methods). Version 4. Sunderland, Massachusetts: Sinauer Associates.

Takahashi T, Sakaguchi K. 1925. Rhizopus zoku no seisan suru san no seishitu ni tsuite (Studies on the acids produced by genus Rhizopus). Nihon Nougeikagaku Kaishi 1:344-355. (In Japanese).

- - - Asai Y. 1926. Rhizopus zoku no seisan suru san no seishitu ni tsuite (Studies on the acids produced by genus Rhizopus). Nihon Nougeikagaku Kaishi 2:396401. (In Japanese).

Takahata N, Nei M. 1985. Gene genealogy and variance of interpopulational nucleotide differences. Genetics 110: 325-344.

Taylor JW, Jacobson DJ, Kroen S, Kasuga T, Geiser D, Hibbett D, Fisher MC. 2000. Phylogenetic species recognition and species concepts in fungi. Fungal Genet Biol 31:21-32.

Thompson JD, Gibson TJ, Plewniak F, Jeanmougin F, Higgins JD. 1997. The Clustal X Windows interface: flexible strategies for multiple sequence alignment aided by quality analysis tools. Nucleic Acid Res 25: $4876-4882$.

Voigt K, Wöstemeyer J. 2001. Phylogeny and origin of 82 zygomycetes from all 54 genera of the Mucorales and Mortierellales based on combined analysis of actin and translation elongation factor EF- $1 \alpha$ genes. Gene 270: 113-120.

Weitzman I, McGough DA, Rinald MG, Della-Latta P. 1996. Rhizopus schipperae, sp. nov., a new agent of zygomycosis. Mycotaxon 59:217-225.

White TJ, Bruns T, Lee S, Taylor J. 1990. Amplification and direct sequencing of fungal ribosomal RNA genes for phylogenetics. In: Innis MA, Gelfand DH, Sninsky JJ, White TJ, eds. PCR protocols: a guide to methods and applications. San Diego: Academic Press. p 315-322.

Yap I, Nelson RJ. 1996. Winboot: a program for performing bootstrap analysis of binary data to determine the confidence limits of UPGMA-based dendrograms. IRRI Discussion Paper 14. Manila, Philippines: International Rice Research Institute.

Yuan G-F, Jong S-C. 1984. A new obligate azygosporic species of Rhizopus. Mycotaxon 20:397-400. 